\title{
Crystallization Behavior of Acetaminophen in Nanopores
}

\author{
Gopalakrishnan T. Rengarajan ${ }^{1}$, Dirk Enke ${ }^{2}$ and Mario Beiner ${ }^{*}, 1$ \\ ${ }^{1}$ Martin-Luther-Universität Halle-Wittenberg, Institut für Physik, 06099 Halle/Saale, Germany \\ ${ }^{2}$ Martin-Luther-Universität Halle-Wittenberg, Institut für Chemie, 06099 Halle/Saale, Germany
}

\begin{abstract}
The influence of nanoconfinement on the crystallization behavior of acetaminophen, a polymorphic drug occurring in three different crystalline forms, is investigated. Differential scanning calorimetry (DSC) and wide angle X-ray scattering (WAXS) data for a series of controlled porous glasses (CPGs) filled with acetaminophen are presented. The results show clearly that (i) the usually inaccessible crystalline form III of acetaminophen can be produced in pores with diameters between 22 and $103 \mathrm{~nm}$ and that (ii) the life time of amorphous acetaminophen is significantly increased in $10 \mathrm{~nm}$ pores. Bulk melting temperature and heat of melting of form III are estimated based on the Gibbs-Thomson equation. The experimental findings are confronted with the predictions of theoretical approaches aimed to describe thermodynamics and crystallization kinetics in nano-sized systems in order to understand the physical background of the observed changes.
\end{abstract}

\section{INTRODUCTION}

The manipulation of the crystallization behavior of substances existing in different polymorphic forms is an important issue in many fields of application [1]. Polymorphic pharmaceuticals are famous examples for the need to control crystallization $[2,3]$. There is a common interest to stabilize unstable crystalline forms of pharmaceuticals since solubility and bioavailibility are usually improved. Even more interesting are amorphous drugs especially if they are stable for a long period of time $[4,5]$. However, this situation is hard to achieve in practice since pharmaceuticals show usually a strong tendency to crystallize. We will show in this paper that one interesting approach to make progress in this field is to confine polymorphic pharmaceuticals in nanoporous host systems.

There are different experimental findings which suggest that nanoporous host systems might be an interesting tool to manipulate the crystallization behavior of pharmaceuticals. On the one hand it has been shown in different studies that the crystallization tendency of simple molecular liquids in nanopores with diameters $<10 \mathrm{~nm}$ is significantly reduced. Liquids which are usually easily crystallizable like benzyl alcohol or orthoterphenyl are not able to crystallize in controlled porous glasses (CPGs) with very small pores [6-8]. Similar behavior has been reported for metals like lead confined in hollow carbon nanotubes [9]. Another finding indicating that nanoconfinement might be useful strategy is that the crystalline structure of nano-sized crystals is often quite different from the situation in the bulk. It is well known that metastable forms of ice grow preferentially in nano-sized water droplets [10]. Recently, first experiments have been reported showing that the crystallization behavior of polymorphic substances is influenced if confined in nanoporous polymers or glass pores having diameters of about $20 \mathrm{~nm}$ $[11,12]$. Similar effects should appear in case of pharmaceuticals.

*Address correspondence to this author at the Universität Halle, Institut für Physik, FG Experimentelle Polymerphysik, Hoher Weg 8, D-06099 Halle/ Saale, Germany; Tel: +49 345 5525350; Fax: +49 345 5527351;

E-mail: beiner@physik.uni-halle.de
A suitable pharmaceutical model system to study the effects of confinement on the crystallization behavior is acetaminophen being a common analgesic and anti-pyretic drug existing in three different crystalline forms [13-16]. The chemical structure of this drug is shown in the insert of Fig. (1a). The commercially used monoclinic form $I$ is the most stable one and has a melting temperature of about 167$169^{\circ} \mathrm{C}$. The orthorhombic form II is metastable and melts at $\sim 156^{\circ} \mathrm{C}[17,18]$. Several preparation procedures have been reported to achieve these two crystalline forms either from solution or from the melt by thermal treatments [13-16]. A third polymorph of acetaminophen - called form III - is reported to be unstable and usually inaccessible in case of bulk samples. This form has been found only in special situations where acetaminophen was confined between glass plates or in thin glass capillaries $[19,20]$. Thus, there is limited information about this polymorph. RAMAN spectra have been reported [19] and first wide angle X-ray scattering data are published recently [20]. However, the investigated form III samples are produced by chance, melting of this crystalline form has never been reported and thermodynamic parameters are basically unknown.

In this paper we report results for host-guest systems where acetaminophen is infiltrated in controlled porous glasses with well defined pore diameters in the range 10-103 $\mathrm{nm}$ and untreated pore walls with high surface energy. We show that nanoconfinement is an efficient method to increase the stability of form III of acetaminophen as well as the life time of the amorphous state. Systematic changes are observed due to confinement giving additional insights into thermodynamics and crystallization kinetics of nano-sized systems.

\section{EXPERIMENTAL}

\section{Materials}

Acetaminophen $\left(\mathrm{C}_{8} \mathrm{H}_{9} \mathrm{NO}_{2}\right.$, insert Fig. (1a)) having a molecular weight of $151.16 \mathrm{~g} \cdot \mathrm{mol}^{-1}$ and a purity $\geq 99 \%$ was obtained from Sigma-Aldrich (CAT No. 103-90-2).

Controlled porous glasses (CPGs) with pore diameters of $10,22,43,60$ and $103 \mathrm{~nm}$ are obtained by leaching of phaseseparated sodium borosilicate glasses containing 70 wt.-\% 
$\mathrm{SiO}_{2}, 23$ wt.- $\% \mathrm{~B}_{2} \mathrm{O}_{3}$ and 7 wt.- $\% \mathrm{Na}_{2} \mathrm{O}[21,22]$. Nanoporous glass monoliths have been prepared in three steps: (i) generation of the desired shape by various techniques of sawing, (ii) heat treatment for phase separation in the temperature range between 530 and $720^{\circ} \mathrm{C}$, (iii) removal of the soluble phase by acid or combined acid/alkaline leaching treatment. Thin platelets having a thickness of about $300 \mu \mathrm{m}$ are produced. The scanning electron microscopy (SEM) image of a controlled porous glass in Fig. (1a) shows that the pores are interconnected and have a sponge-like morphology. The pore size distribution is narrow (Fig. 1b) and the porosity is usually about $50 \%$. Further details about the textural properties of all CPGs used in our study as host systems are summarized in Table $\mathbf{1}$.
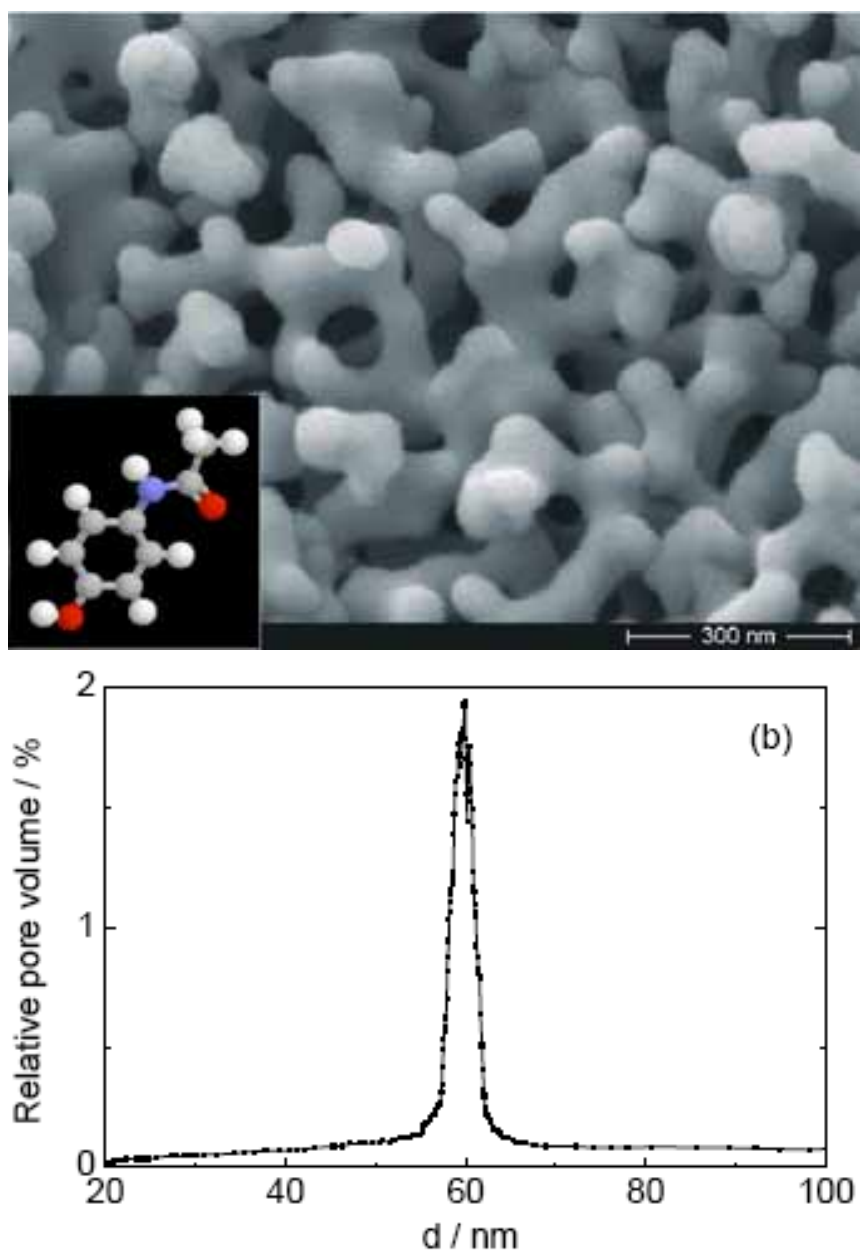

Fig. (1). (a) Scanning electron microscopy image of a controlled porous glass used as host system. Insert: chemical structure of acetaminophen (gray: carbon; red: oxygen; white: hydrogen; blue: nitrogen). (b) Pore size distribution for the CPG having an average diameter of $60 \mathrm{~nm}$ as obtained from mercury porosimetry measurements.

\section{Sample Preparation}

The thin CPGs platelets were carefully dried in the vacuum oven at $180^{\circ} \mathrm{C}$ for 2 hours to remove entrapped moisture. The pre-dried glass substrates are filled by immersing it in molten acetaminophen maintained at a temperature of $180^{\circ} \mathrm{C}$. Then, the filled CPG platelets are taken out and cooled to room temperature in the presence of an acetaminophen surface layer, which was later carefully removed using a scalpel. Small pieces of the filled CPG having a mass of about $10 \mathrm{mg}$ were encapsulated in aluminum pans $(10 \mu \mathrm{l})$ for subsequent DSC measurements.

Table 1. Textural Properties of the CPGs Used as Host Systems ${ }^{\mathrm{a}}$

\begin{tabular}{|c|c|c|c|}
\hline $\boldsymbol{d}(\mathbf{n m})$ & $\boldsymbol{\mu}\left(\mathbf{m}^{\mathbf{2}} \mathbf{g}^{\mathbf{- 1}}\right)$ & $\boldsymbol{\nu}\left(\mathbf{c m}^{\mathbf{3}} \mathbf{g}^{\mathbf{- 1}}\right)$ & $\mathbf{P}(\boldsymbol{\%})$ \\
\hline \hline 10 & 76 & 0.20 & 31 \\
\hline 22 & 81 & 0.40 & 47 \\
\hline 43 & 36 & 0.36 & 44 \\
\hline 60 & 30 & 0.43 & 49 \\
\hline 103 & 30 & 0.53 & 54 \\
\hline
\end{tabular}

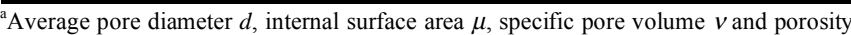
$P$ values are determined based on mercury porosimetry measurements.

\section{DSC Measurements}

PerkinElmer Pyris Diamond and DSC 7 instruments have been used for Differential Scanning Calorimetry (DSC) measurements. A standard program was applied to all samples. (i) First heating scans on samples crystallized in the presence of an acetaminophen surface layer during the preparation have been performed with a rate of $+10 \mathrm{~K} / \mathrm{min}$. The scan was started after cooling the sample to $-40^{\circ} \mathrm{C}$ and ended at $180^{\circ} \mathrm{C}$ well above the melting temperature of form I of acetaminophen. (ii) Then the samples are held at $180^{\circ} \mathrm{C}$ for $3 \mathrm{~min}$ and quenched to $-40^{\circ} \mathrm{C}$ with a nominal DSC cooling rate of $-200 \mathrm{~K} / \mathrm{min}$ corresponding to real rates of at least $-100 \mathrm{~K} / \mathrm{min}$. This produces nearly $100 \%$ glassy acetaminophen in the pores. Second heating scans were performed in the temperature range -40 to $180^{\circ} \mathrm{C}$. (iii) Afterwards the samples were held again at $180^{\circ} \mathrm{C}$ for $3 \mathrm{~min}$ and quenched to $-40^{\circ} \mathrm{C}$, reheated to $T_{\mathrm{c}}=80^{\circ} \mathrm{C}$ at a rate of $10 \mathrm{~K} / \mathrm{min}$ and annealed at $T_{\mathrm{c}}$ for $2 \mathrm{~h}$ with the aim to achieve $100 \%$ crystalline samples. Finally the system was cooled to $-40^{\circ} \mathrm{C}$ and a third heating scan to $180^{\circ} \mathrm{C}$ was performed at a rate of $10 \mathrm{~K} / \mathrm{min}$.

The melting temperatures $T_{\mathrm{m}}$ given in this paper correspond to the maximum of the melting peak. Heats of melting $\Delta H_{m}$ are calculated from the area of the melting peaks. The baselines for these calculations are estimated based on a linear extrapolation of the heat capacity in the molten state.

\section{X-Ray Scattering Measurements}

The wide angle X-ray scattering (WAXS) data shown in this paper are measured in $\theta-2 \theta$ geometry with $\mathrm{CuK} \alpha$ radiation having a wavelength of $\lambda=1.542 \mathrm{~nm}$. The measurements have been performed at room temperature. The average measurement time per data point is about $30 \mathrm{~s}$.

\section{RESULTS}

DSC heating scans for acetaminophen filled in CPGs with $43 \mathrm{~nm}$ pores after different thermal treatment are presented in Fig. (2). The first heating scan performed on a sample crystallized during cooling in the presence of an acetaminophen surface layer shows a major melting peak near $160^{\circ} \mathrm{C}$ slightly below the melting temperature of form I in bulk acetaminophen $\left(T_{\mathrm{m}, \mathrm{I}}^{\infty}=167-169^{\circ} \mathrm{C}\right)$ [17,18]. This peak indicates melting of the monoclinic form I of acetaminophen in the pores as seen in room temperature WAXS pattern for identically prepared samples (Fig. 3). The fact 
that there is no cold crystallization peak in the first DSC heating scan shows that the as-prepared samples are nearly $100 \%$ crystalline before heating. The small melting peak near $167^{\circ} \mathrm{C}$ indicates melting of a small fraction of acetaminophen remaining at the surface after mechanical cleaning. The second heating scan in Fig. (2) shows that the confined drug is practically $100 \%$ amorphous (glassy) after quenching the sample rapidly from $180^{\circ} \mathrm{C}$ to $-40^{\circ} \mathrm{C}$. During reheating the thermal glass transition (insert Fig. (2)) occurs near the bulk glass temperature $\left(T_{\mathrm{g}}=24^{\circ} \mathrm{C}\right)$ [23] of acetaminophen and a significant cold crystallization peak appears between $75^{\circ} \mathrm{C}$ and $100^{\circ} \mathrm{C}$. Cold crystallized acetaminophen in $43 \mathrm{~nm}$ pores melts near $135^{\circ} \mathrm{C}$ slightly below the speculative melting temperature of form III $\left(\approx 139^{\circ} \mathrm{C}\right)$ which has been mentioned in a paper by Giordano et al. [24] dealing with acetaminophen/methylcellulose mixtures. X-ray scattering data for identically prepared $\left(T_{\mathrm{c}}=80^{\circ} \mathrm{C}, t_{\mathrm{c}}=2 \mathrm{~h}\right)$ hostguest systems measured at room temperature support the existence of form III crystals in filled CPGs. The obtained WAXS pattern (Fig. 3) is comparable to that one reported recently by Peterson et al. [20] for form III of acetaminophen grown by chance in thin glass capillaries. The tiny melting peak at $156^{\circ} \mathrm{C}$ in the second heating scan in Fig. (2) indicates melting of form II crystals on the surface. This behavior corresponds to that of bulk acetaminophen crystallized starting from the glassy state [23]. The third heating scan measured after quenching and $2 \mathrm{~h}$ isothermal crystallization at $T_{\mathrm{c}}=80^{\circ} \mathrm{C}$ is similar to the second scan. The only difference is the absence of a cold crystallization peak since the crystallization process is already finalized at $T_{\mathrm{c}}$ before the third heating scan is started. Obviously, form III of acetaminophen is also growing during isothermal crystallization at $80^{\circ} \mathrm{C}$ in $43 \mathrm{~nm}$ pores.

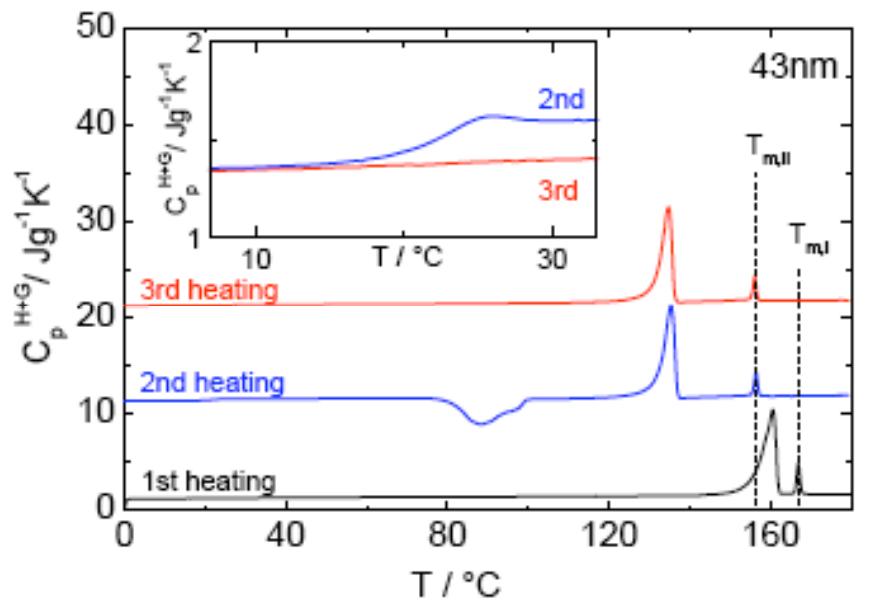

Fig. (2). DSC heating scans for acetaminophen in a CPG with 43 $\mathrm{nm}$ pores. A first heating scan performed on an as-prepared sample, second heating after quenching and third heating after quenching and isothermal crystallization at $80^{\circ} \mathrm{C}$ for $2 \mathrm{~h}$ are compared (cf. experimental section). The curves are vertically shifted by $10 \mathrm{Jg}^{-1} \mathrm{~K}^{-1}$ for the sake of clarity. The insert shows a zoom of the glass transition region. Heat capacities for the host-guest system $C_{\mathrm{p}}{ }^{\mathrm{H}+\mathrm{G}}$ are plotted. The bulk melting temperatures of form II $\left(T_{\mathrm{m}, \mathrm{II}}{ }^{\infty}=156^{\circ} \mathrm{C}\right)$ and form $\mathrm{I}\left(T_{\mathrm{m}, \mathrm{I}}^{\infty}=167^{\circ} \mathrm{C}\right)$ are indicated by dotted lines.

First and third heating scans for acetaminophen in CPGs with different pore diameters between 22 and $103 \mathrm{~nm}$ are compared in Fig. (4). In the first heating scans (Fig. 4a) melting of form I crystals is always seen. Depending on the pore diameter the crystals melt at temperatures between $153^{\circ} \mathrm{C}$ in case of $22 \mathrm{~nm}$ pores and $165^{\circ} \mathrm{C}$ in case of $103 \mathrm{~nm}$ pores. The decrease of the melting temperature with decreasing pore diameter is due to melting point depression in case of nano-sized crystals. Note, that the small melting peak at $T_{\mathrm{m}, \mathrm{I}}^{\infty} \approx 167^{\circ} \mathrm{C}$ does not shift with the pore diameter consistent with idea that this is a small fraction of acetaminophen remaining on the surfaces. The third heating scans (Fig. 4b) performed on quenched and isothermally crystallized samples indicate that annealing at $80^{\circ} \mathrm{C}$ for $2 \mathrm{~h}$ gives almost $100 \%$ crystalline samples. There is no glass transition, no cold crystallization peak and the heat of melting is always similar to the values obtained in the first scans. Whether or not there is a very thin immobilized (non-crystallizable) interfacial layer like in other host guest systems [25-27] remains open at this point but might be an interesting point for further investigations.

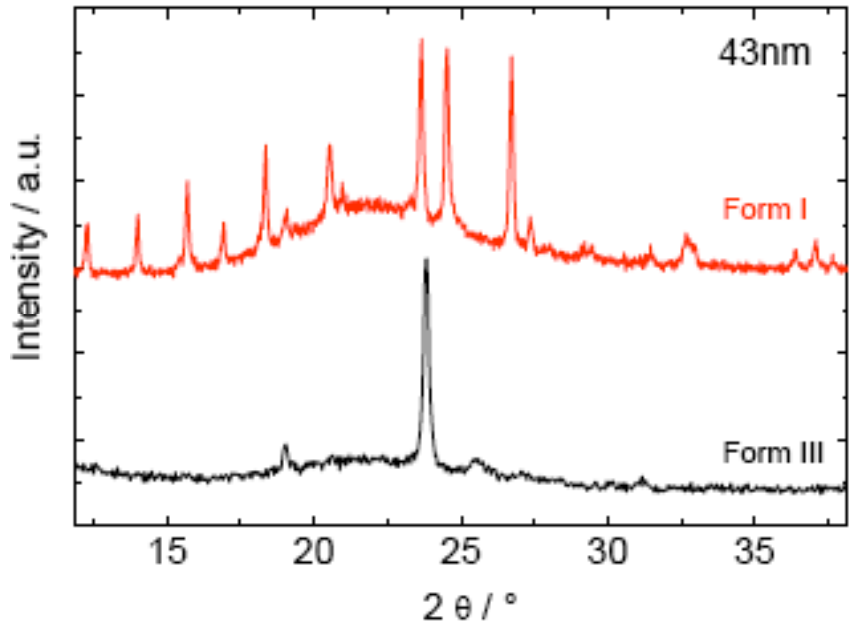

Fig. (3). Wide angle X-ray scattering curves for acetaminophen confined in CPGs with $43 \mathrm{~nm}$ pores measured at room temperature. The upper curve for an as-prepared sample (= first heating) shows the pattern of form I and the lower curve for a sample after $2 \mathrm{~h}$ isothermal crystallization at $80^{\circ} \mathrm{C}$ (= third heating) that of form III crystals (for comparison see Ref. [20]). The broad halo in both curves is due to contributions of the porous glass. The curves are vertically shifted.

In the third heating scans (Fig. $\mathbf{4 b}$ ) melting of form III is seen for all pore diameters $d$. The melting temperature decreases with decreasing diameter $d$ from $140^{\circ} \mathrm{C}$ in case of $103 \mathrm{~nm}$ pores to $125^{\circ} \mathrm{C}$ in $22 \mathrm{~nm}$ pores. In all cases a small fraction of surface material melts as form II near $156^{\circ} \mathrm{C}$. For the sample with $103 \mathrm{~nm}$ pores an additional melting peak around $152^{\circ} \mathrm{C}$ is observed. This peak indicates melting of form II crystals under confinement. In general, the fraction of form II crystals which melts seems to increase if higher crystallization temperatures and pores diameters $>100 \mathrm{~nm}$ are used [28] indicating a transition to bulk behavior where form II of acetaminophen is melting exclusively under similar conditions [23]. Speculatively, form III crystals, which may also grow in bulk acetaminophen at low temperatures, are able to undergo a solid-solid transformation [14,19,23] into form II near $120^{\circ} \mathrm{C}$ in the bulk before melting occurs at $156^{\circ} \mathrm{C}$. This would explain why melting of form III is not seen in bulk samples. 

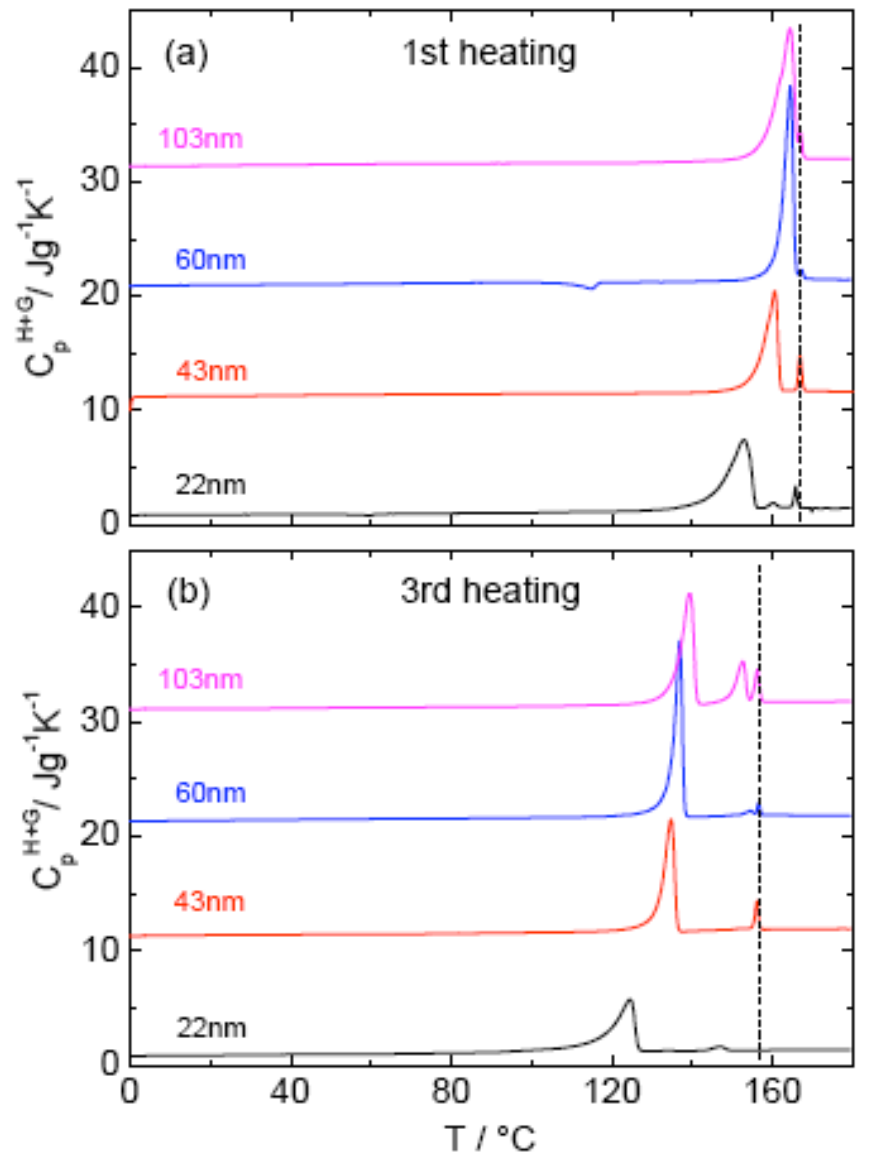

Fig. (4). DSC heating scans for acetaminophen in CPGs with different pore diameters. Part (a) shows first heating scans on asprepared samples and part (b) third heating scans performed after crystallizing the sample at $80^{\circ} \mathrm{C}$ for $2 \mathrm{~h}$. Form I is melting in the first case while melting of form III is seen in the latter case. The dotted lines in parts (a) and (b) indicate the bulk melting temperatures of form I $\left(T_{\mathrm{m}, \mathrm{I}}^{¥} \approx 167^{\circ} \mathrm{C}\right)$ and form II $\left(T_{\mathrm{m}, \mathrm{II}}^{¥} \approx 156^{\circ} \mathrm{C}\right)$, respectively. The curves are vertically shifted by $10 \mathrm{Jg}^{-1} \mathrm{~K}^{-1}$.

The dependence of the melting temperatures on the pore diameter $d$ is shown for three different crystalline forms of acetaminophen in Fig. (5). The obtained reduction of $T_{\mathrm{m}}$ with decreasing pore diameter is well described by a GibbsThomson equation having in case of cylindrical pores the form $[6,12]$

$T_{\mathrm{m}}(d)=T_{\mathrm{m}}{ }^{\infty} \cdot\left[1-4 \cdot \sigma_{\mathrm{cl}} /\left(d \cdot \Delta H_{\mathrm{m}} \cdot \rho_{\mathrm{c}}\right)\right]$

where $T_{\mathrm{m}}{ }^{\infty}$ is the melting temperature of the bulk corresponding to infinitively thick crystals, $\Delta H_{\mathrm{m}}$ the heat of melting, $\sigma_{\mathrm{cl}}$ the surface tension between crystalline and liquid phase and $\rho_{\mathrm{c}}$ the crystal density.

For all three crystalline forms of acetaminophen a nearly linear dependence of $T_{\mathrm{m}}$ on the reciprocal pore diameter $1 / d$ is obtained. The slopes $s_{\mathrm{I}}, s_{\mathrm{II}}$ and $s_{\mathrm{III}}$ are also similar indicating that the difference in $T_{\mathrm{m}}{ }^{\infty} \cdot \sigma_{\mathrm{cl}} /\left(\Delta H_{\mathrm{m}} \cdot \rho_{\mathrm{c}}\right)$ between the different forms is not dramatic.

An extrapolation of the melting temperatures of forms I and II from small pore diameters to infinitely thick crystals $(d \rightarrow \infty)$ yields the well known bulk melting temperatures of form I and form II of acetaminophen as indicated by the half-filled symbols in Fig. (5). Analogously, the extrapola- tion of the melting temperatures of form III crystals in pores with different diameter gives the bulk melting temperature of this usually inaccessible form which has not been reported to our knowledge so far. We obtain $T_{\mathrm{m}, \mathrm{III}}{ }^{\infty}=143^{\circ} \mathrm{C}$.

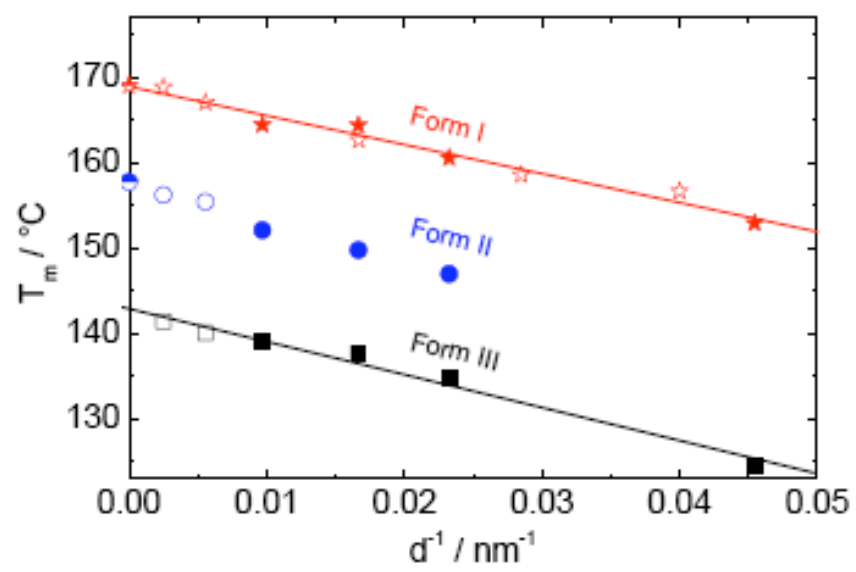

Fig. (5). Gibbs-Thomson plot for three different crystalline forms of acetaminophen showing the melting temperatures $T_{\mathrm{m}}$ as function of the reciprocal pore diameter $(1 / d)$. Data for acetaminophen confined in CPGs (solid symbols) and mesoporous alumina (open symbols) [28] as well as bulk melting temperatures (half-filled symbols) [17, 18] are included. The lines are linear fits to the data for forms I and III based on Eq.(1).

Note, that a similar melting point depression like in CPGs is also obtained if acetaminophen is confined in pores of mesoporous alumina [28]. The melting temperatures taken from first scans, where form I melts in the pores, are shown for comparison in Fig. (5). This result is not so surprising since the contact angles measured at $170^{\circ} \mathrm{C}$ for acetaminophen on glass $\left(\approx 20^{\circ}\right)$ and acetaminophen on alumina $\left(\approx 27^{\circ}\right)$ are comparable showing that the surface interaction is similar in both types of host-guest systems. On the other hand this result supports the relatively strong influence of the pore diameter on the melting temperature in case of acetaminophen which seems to be large compared to that for many other small molecule liquids confined in CPGs [7].

Another thermodynamic parameter which can be estimated for the first time based on the experimental data presented in this paper is the heat of melting of form III of acetaminophen, $\Delta H_{\mathrm{m}, \mathrm{III}}$. This can be done in two different ways: The first version is based on the Gibbs-Thomson equation (Eq. (1)). Fitting the data for form I and form III in the Gibbs-Thomson plot (Fig. 5) one gets the slopes $s_{\mathrm{I}}=340$ $\mathrm{K} \cdot \mathrm{nm}$ and $s_{\mathrm{III}}=386 \mathrm{~K} \cdot \mathrm{nm}$, respectively. Based on these values and $\Delta H_{\mathrm{m}, \mathrm{I}} \approx 186.1 \mathrm{Jg}^{-1}[17,18]$ one can estimate the heat of melting according to

$\Delta H_{\mathrm{m}, \mathrm{III}}=T_{\mathrm{m}, \mathrm{III}}^{\infty} \cdot s_{\mathrm{I}} \cdot \Delta H_{\mathrm{m}, \mathrm{I}} /\left(s_{\mathrm{III}} \cdot T_{\mathrm{m}, \mathrm{I}}^{\infty}\right)$

assuming that the ratio $\sigma_{\mathrm{cl}} / \rho_{\mathrm{c}}$ is identical for form I and form III. This gives an estimated heat of fusion $\Delta H_{\mathrm{M}, \mathrm{III}}$ of about $154 \pm 15 \mathrm{Jg}^{-1}$. The second version is based on a combination of first and third heating scans as shown in Fig. (4). Assuming that the degree of crystallinity is $100 \%$ in both cases and using the heat of fusion $\Delta H_{\mathrm{M}, \mathrm{I}}$ of form I as reported the literature one can estimate the mass $m$ of acetaminophen in a given sample based on the simple relationship

$m=\Delta h_{\mathrm{m}, \mathrm{I}} / \Delta H_{\mathrm{m}, \mathrm{I}}$ 
with $\Delta h_{\mathrm{m}, \mathrm{I}}$ being the experimentally obtained heat of melting from the first scan. Starting from this mass $m$ and using the experimental $\Delta h_{\mathrm{M}, \mathrm{III}}$ value from the third scan on the same sample one can estimate the heat of melting for form III. We obtain $\Delta H_{\mathrm{m}, \mathrm{III}}=165-188 \mathrm{Jg}^{-1}$ with a certain variation depending on the pore diameter used. This value is in crude agreement with the $\Delta H_{\mathrm{M}, \mathrm{III}}$ value obtained using the other method described above. Note, that the second approach neglects possible changes of $\Delta H_{\mathrm{m}}$ in very small pores ("pore size dependence of $\Delta H_{\mathrm{m}}$ ") [6] which would lead to modifications in the estimated $\Delta H_{\mathrm{m}, \mathrm{III}}$ value.

Additional information about the influence of nanoconfinement on the crystallization kinetics is provided in Fig. (6). Isothermal crystallization of acetaminophen in 103 $\mathrm{nm}$ pores is studied at different temperatures $T_{\mathrm{c}}$. The sample is held for a specific time $t_{\mathrm{c}}$ at $T_{\mathrm{c}}$ and then reheated to determine heat of melting $\Delta h_{\mathrm{M}, \mathrm{III}}$ and degree of crystallinity $D_{\mathrm{c}}$. The isothermal crystallization temperature was always approached from the glassy state obtained by quenching molten acetaminophen in the pores rapidly to $-40^{\circ} \mathrm{C}$ with a cooling rate of at least $-100 \mathrm{~K} / \mathrm{min}$ (nominal DSC cooling rate -200 $\mathrm{K} / \mathrm{min}$ ). Fig. (6a) shows that the crystallization is faster at higher temperatures $T_{\mathrm{c}}$ indicating that the mobility is dominating the crystallization kinetics of acetaminophen in this temperature range. A similar trend is observed for bulk acetaminophen [23]. A comparison of temperaturedependent half-times $\tau_{1 / 2}$ for acetaminophen in $103 \mathrm{~nm}$ pores with those for bulk samples is presented in Fig. (6b). This plot shows that the crystallization in interconnected $103 \mathrm{~nm}$ pores is about five times slower than in the bulk while the temperature dependence of $\tau_{1 / 2}$ is not too different. Note, that the transformation interval in the crystallization isotherms (Fig. 6a) is only slightly broader than in case of bulk acetaminophen. Expressed in terms of Avrami coefficients [29] one gets values between $n=1.5$ for $T_{\mathrm{c}}=70^{\circ} \mathrm{C}$ and $n=2.5$ for $T_{\mathrm{c}}=50^{\circ} \mathrm{C}$ compared to $n \sim 3$ as reported for bulk acetaminophen [23]. Extremely broad transformation intervals and Avrami exponents near $n=1$ as usually observed in case of homogeneous nucleation [30,31] do not appear. The smaller $n$ values for acetaminophen confined in CPGs might be due to changes in the growth mechanism since the crystals must fill small cylindrical pores with a sponge-like morphology. There are indications for a slight decrease of $n$ with temperature which are not understood so far.

Another interesting effect of nanoconfinement on the crystallization behavior is seen for the CPGs with the smallest pores in our study having a diameter of only $10 \mathrm{~nm}$. The crystallization of acetaminophen is strongly depressed and the guest system is remaining in the amorphous state during isothermal crystallization at $80^{\circ} \mathrm{C}$ for $2 \mathrm{~h}$. Crystalline acetaminophen is only seen in the first heating scan, i.e. if crystallized during the preparation under the influence of a thick acetaminophen surface layer (Fig. 7). In this case form I is melting at temperatures below $140^{\circ} \mathrm{C}$ as expected based on the results for the other host guest systems. In all later heating scans melting peaks are missing, i.e. acetaminophen in $10 \mathrm{~nm}$ pores crystallizes neither during cooling with rate of $10 \mathrm{~K} / \mathrm{min}$ and reheating with $+10 \mathrm{~K} / \mathrm{min}$ (second heating) nor during isothermal crystallization at $80^{\circ} \mathrm{C}$ for $2 \mathrm{~h}$ (third heating). Note, that the thermal glass transition occurs at a temperature around $5^{\circ} \mathrm{C}$ significantly below the bulk glass temperature $\left(T_{\mathrm{g}}=24^{\circ} \mathrm{C}\right)$. Whether this is a geometrical confine- ment effect, due to changes in density or an indication for a certain moisture content in the host system needs further investigation. The observation that acetaminophen in untreated glass pores having a diameter of $10 \mathrm{~nm}$ is not easily crystallizable, however, is untouched by this open question and an interesting finding since methods to stabilize amorphous pharmaceuticals are important for their application [32].
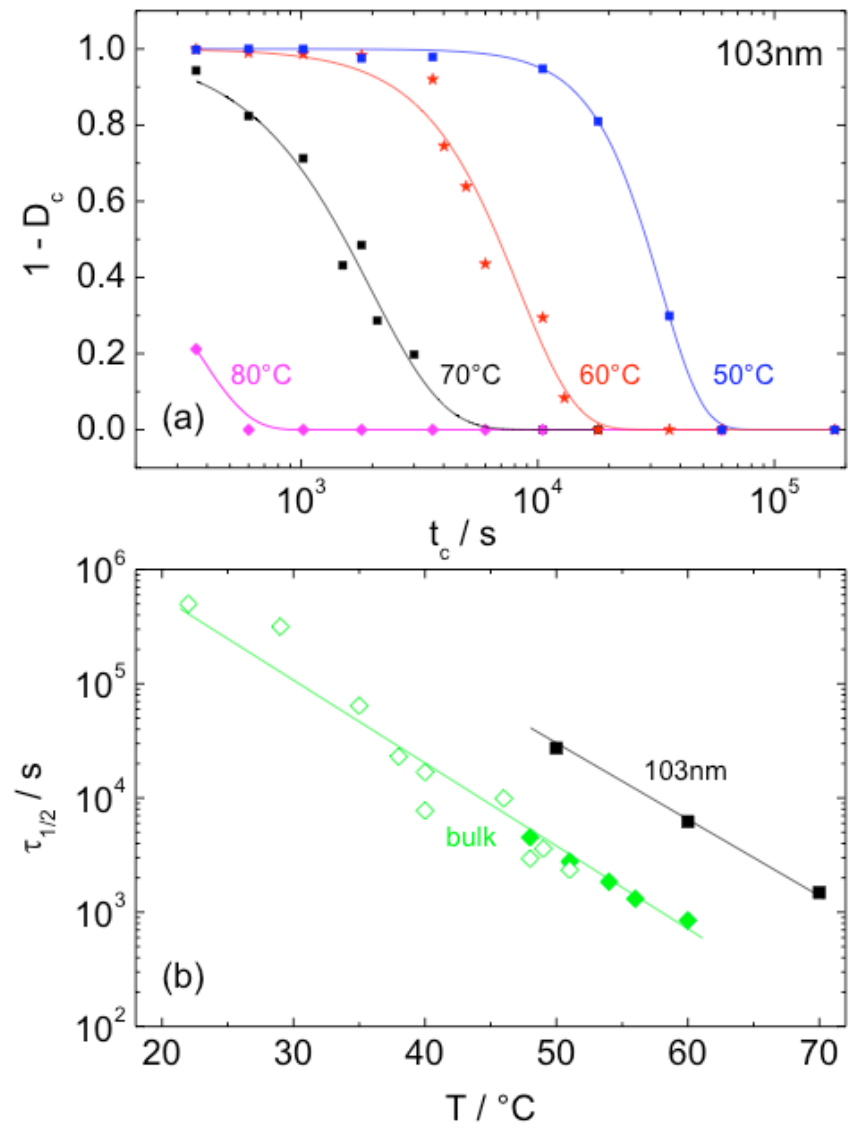

Fig. (6). (a) Crystallization kinetics of acetaminophen in a CPG with $103 \mathrm{~nm}$ pores. $D_{\mathrm{c}}$ is calculated based on the heat of melting $\Delta h_{\mathrm{m}}$ in DSC heating scans performed after isothermal crystallization for a time $t_{\mathrm{c}}$ at $T_{\mathrm{c}}$ normalized by a common factor $\Delta h_{\mathrm{m} \text {,max }}$. The solid lines are fits using the Avrami equation $1-D_{\mathrm{c}}=\exp (-\mathrm{kt})^{\mathrm{n}}$ with $n$ being the Avrami exponent and $k$ a crystallization rate [29]. (b) Temperature-dependent half-times $\tau_{\mathrm{c}}$ (time to reach $50 \%$ crystallinity) for confined and bulk acetaminophen. Data for acetaminophen in $103 \mathrm{~nm}$ pores $(\boldsymbol{\square})$ are taken from isotherms as shown in part (a), results for bulk acetaminophen from dielectric spectroscopy $(\diamond)$ and DSC $(\bullet)$ [23] are shown for comparison.

\section{DISCUSSION}

The main question that arises based on the presented results is why less stable crystalline forms and amorphous state are observed if we confine acetaminophen in nanometersized pores of untreated CPGs. There are at least three effects which can influence the crystallization behavior in small pores: (i) The equilibrium thermodynamics changes due to surface energy contributions to the total free energy. In nanoscopic systems this can cause the stabilization of phases which are in unstable in the bulk and can influence phase transition temperatures. (ii) The nucleation mechanism can be influenced due to space restrictions and separation 
into sub-volumes. (iii) The crystallization kinetics can slow down since immobilized surface layers with a thickness of a few nanometers are seemingly a common feature at pore walls with high surface energy. While the first phenomenon (i) is a thermodynamic equilibrium effect the two other phenomena (ii,iii) will change the kinetics and influence nonequilibrium aspects of the crystallization process. We will consider below the relevance of these effects for our systems.

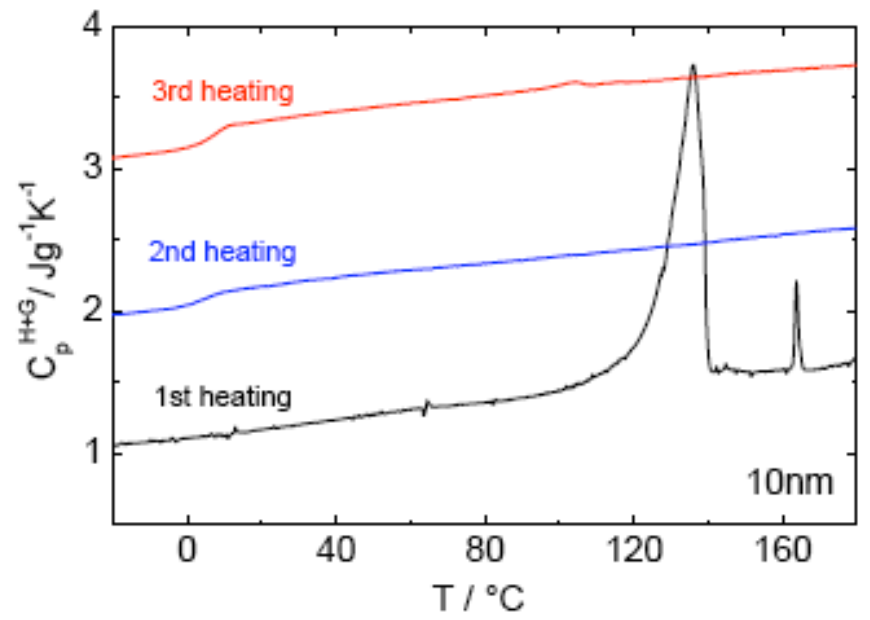

Fig. (7). DSC heating scans for acetaminophen in CPGs with 10nm pores. First, second and third heating scans according to the program described in the experimental section are shown. The curves are vertically shifted by $1 \mathrm{Jg}^{-1} \mathrm{~K}^{-1}$ for the sake of clarity.

The stabilization of conventionally unstable states in nanoscopic systems has been explained using thermodynamic arguments in the past. The occurrence of amorphous metals [33] and the vanishing crystallization of molecular liquids [34] in small nanopores has been discussed in this way. Based on the balance of energetic contributions of surface and volume to the total free energy a critical diameter $d^{*}$ required to get a crystal that tends to grow in a cylindrical pore can be estimated [33,34].

$d^{*}=4 T_{m}{ }^{\infty} \cdot \sigma_{\mathrm{cl}} /\left[\Delta H_{\mathrm{m}} \cdot \rho_{\mathrm{c}} \cdot\left(T_{m}{ }^{\infty}-T\right)\right]$

with $\Delta H_{\mathrm{m}}$ being the heat of melting, $\sigma_{\mathrm{cl}}$ the crystal-toliquid surface energy and $T_{\mathrm{m}}{ }^{\infty}$ the bulk melting temperature. This gives usually $d^{*}$ values in the range of a few nanometers [34]. We have estimated for form III crystals at $80^{\circ} \mathrm{C} \mathrm{a}$ critical diameter of about $6.2 \mathrm{~nm}$ using the slope $s_{\mathrm{III}}$ in the Gibbs-Thomson plot (Fig. 5). In pores having a smaller diameter form III crystals should not be able to grow. The stability of metastable crystalline forms (cubic ice) in nanosized water droplets has been explained using similar thermodynamic arguments [10]. The critical diameter $d_{\mathrm{A} / \mathrm{B}}$ required for a transition between two polymorphic forms $\mathrm{A}$ and $\mathrm{B}$ can be calculated for cylindrically shaped crystals from $[10,35]$.

$d_{\mathrm{A} / \mathrm{B}}=4 \cdot \sigma_{\mathrm{A} / \mathrm{B}} /\left[\Delta H_{\mathrm{A} / \mathrm{B}} \cdot \rho_{\mathrm{c}}\right]$

with $\Delta H_{\mathrm{A} / \mathrm{B}}$ being the heat of transformation, $\sigma_{\mathrm{A} / \mathrm{B}}$ the relevant surface energy and $\rho_{c}$ the density. The usually metastable crystalline form B should be the stable state below this critical size $d_{\mathrm{A} / \mathrm{B}}$. Another consequence of thermodynamic changes in nano-sized systems can be a shift of the transition temperatures between different crystalline forms depending on the pore diameter. Pressure-dependent changes in the phase behavior of polymorphic materials have been reported frequently [1].

A closer look on our experimental results shows that thermodynamic changes alone are not sufficient to describe the situation in the investigated host-guest systems. The $d^{*}$ values at $T<80^{\circ} \mathrm{C}$ are smaller than $10 \mathrm{~nm}$. Accordingly, a significant crystalline fraction can be also detected in $10 \mathrm{~nm}$ pores after long storage times (a few weeks) at room temperature as well as in the first heating scan (Fig. 7) where crystals can grow from nuclei located in a thick acetaminophen surface layer. In larger pores $(d>22 \mathrm{~nm})$ either form III or form I crystals do grow depending on the program. Both crystalline forms persist at room temperature for practically infinite time (several weeks) although only one crystalline form can be thermodynamically stable under these conditions. Subsequent heating scans show melting of that form which was grown initially. Obviously, thermodynamic and kinetic aspects have to be considered in order to understand the situation.

Starting point of our current interpretation is that form III crystals are thermodynamically stable at low temperatures since this form is obviously growing in our crystallization experiments near $80^{\circ} \mathrm{C}$. A solid-solid transition between form III and form II near $120^{\circ} \mathrm{C}$ has been discussed for bulk acetaminophen by several authors $[14,19,23,39]$ and we have observed significant amounts of form II in host-guest systems which are isothermally crystallized at $T>120^{\circ} \mathrm{C}$ [32]. A solidsolid transition of form II to form I at temperatures around $140^{\circ} \mathrm{C}$ has been reported for bulk acetaminophen [18] and growth of form I in nanopores at similar temperatures is indicated in recent experiments. This could explain why either form I or form III crystals can grow if different timetemperature programs are applied (Fig. 4). The crystallization temperature during the cooling step of the sample preparation will be much higher than $80^{\circ} \mathrm{C}$ where the isothermal crystallization experiments are usually performed.

This alone, however, can not explain why form III is melting in nanopores. Changes in the crystallization kinetics must be considered in order to understand this behavior which has never been observed for bulk acetaminophen. The main difference between bulk and confined liquid might be that the transformation of form III into form II near $120^{\circ} \mathrm{C}$ takes in nanopores much longer than in the bulk. This could be due to strong surface interaction resulting in a slower transformation process and hindered nucleation. At the walls of untreated CPGs hydrogen bonds can be formed and immobilized surface layers are often reported under similar conditions [25-27]. Hydrogen bonds between the acetaminophen molecules might be the reason why regions far away from the interface are also affected. Moreover, the nucleation of more stable crystalline forms may require the formation of larger and more complex nuclei which can not easily grow in nanopores [36]. Speculatively, a combination of these effects leads to situations where the system is trapped in a thermodynamically unstable state for long times. Note, that this should not be unique for our system since intermediate crystalline states should be always passed during the formation of a stable crystalline form in polymorphic materials according to Ostwald's rule proposed already in the 1890's [37].

The increased life time of amorphous acetaminophen in nanopores might also be due to interfacial regions with slower 
dynamics and due to changes in the nucleation behavior. The nucleation process could be affected by the confinement although the pores in CPGs are interconnected and not isolated like in classical experiments showing homogeneous nucleation. Nuclei in cylindrical pores of CPGs could be (i) less efficient since crystal growth is basically restricted to one direction [38], (ii) the formation of homogeneous nuclei could be hindered by immobilized surface layers and (iii) the number of heterogeneous nuclei might be reduced since larger impurities do not fit into the pores. The latter effects would lead to a smaller number of accessible nuclei and could partly explain the gradual increase of the crystallization time with decreasing pore diameter. Note, that the glass walls do not nucleate the crystallization of acetaminophen. The relatively large Avrami coefficients for acetaminophen in $103 \mathrm{~nm}$ pores $(1.5<n<2.5)$ indicate that most of the nuclei are not formed by homogeneous nucleation at the investigated crystallization temperatures $T_{\mathrm{c}}$ since values $n \leq 1$ are usually obtained in such cases $[30,31]$.

Although the discussed picture is only a hypothesis so far it might be a suitable basis for further experiments aimed to understand changes in the crystallization behavior of polymorphic drugs under confinement. The importance of the discussed changes in the kinetics should be checked based on long-term experiments aimed to get more information about the equilibrium situation. However, this can be very complicated in detail since a delicate balance of competing effects can exist in our systems like in many other cases where liquids are confined in nanoporous host systems.

\section{CONCLUSIONS}

Summarizing the results of our investigations on acetaminophen confined in controlled porous glasses (CPGs) we can conclude that the crystallization behavior of this polymorphic drug in nanopores is significantly different from that of bulk samples. Form III of acetaminophen is melting in pores with diameters between $22 \mathrm{~nm}$ and $103 \mathrm{~nm}$ if cold crystallized at low temperatures around $80^{\circ} \mathrm{C}$ starting from the glassy state while form I is melting if acetaminophen is crystallized in the presence of a thick acetaminophen surface layer starting from the molten state. The size dependence of the melting temperature of the different crystalline forms is quantified and can be used to estimate for the first time bulk melting temperature $\left(T_{\mathrm{m}, \mathrm{III}}{ }^{\infty}=143^{\circ} \mathrm{C}\right)$ and heat of melting $\left(\Delta H_{\mathrm{m}, \mathrm{III}}=154 \pm 15 \mathrm{Jg}^{-1}\right)$ of form III of acetaminophen. If acetaminophen is confined in untreated CPGs with $10 \mathrm{~nm}$ pores the life time of the amorphous state is increased. The physical reasons for these changes are not finally understood but there are clear indications that thermodynamic aspects and changes in the crystallization kinetics in pores with high surface energy are important. In any case nanoconfinement seems to be an interesting strategy to manipulate the crystallization behavior of polymorphic drugs and to learn more about the thermodynamics in nano-sized systems as well as early stages of crystallization.

\section{ACKNOWLEDGEMENT}

We thank G.P. Johari for bringing pharmaceuticals to our attention, Ch. Eisenschmidt for X-ray scattering measurements, E. Hempel for assistance with the DSC as well as M. Steinhart, S. Pankaj, K. Schröter and Th. Thurn-Albrecht for helpful discussions. Financial support by the state SachsenAnhalt in the framework of the research cluster "Nanostructured materials" is acknowledged.

\section{REFERENCES}

[1] Bernstein, J. Polymorphism in Molecular Crystals, Oxford Scientific: Oxford, 2002.

[2] Polymorphism in Pharmaceutical Solids; (Ed.: Brittain, H.G.) Marcel Dekker Inc: New York, 1999.

[3] Polymorphism in the pharmaceutical industry (Ed.: Hilfiker, R),Wiley-VCH: Weinheim, 2006.

[4] Hancock, B.C.; Zografi, G. J. Pharm. Sci., 1997, 86, 1.

[5] Roberts, C.J.; DeBenedetti P.G. AIChE J., 2002, 48, 114.

[6] Jackson, C. L.; McKenna, G. B. J. Chem. Phys., 1990, 93, 9002.

[7] Alcoutlabi, M.; McKenna, G. B. J. Phys. Condens. Matter., 2005, 17 , 461.

[8] Alba-Simionesco, C.; Coasne, B.; Dosseh, G.; Dudziak, G.; Gubbins, K. E.; Radhakrishnan, R.; Sliwinska-Bartkowiak, M. J. Phys. Condens. Matter, 2006, 18, R15.

[9] Ajayan, P.M.; Iijima, S. Nature, 1993, 361, 333.

[10] Johari, G.P. J. Chem. Phys., 2005, 122, 194504.

[11] Ha, J. M.; Wolf, J. H.; Hillmyer, M. A.; Ward, M. D. J. Am. Chem. Soc., 2004, 126, 3382.

[12] Ha, J. M.; Hillmyer, M. A.; Ward, M. D. J. Phys. Chem. B, 2005, 109, 1392.

[13] Espeau, P.; Ceolin, R.; Tamarit, J. L.; Perrin, M. A.; Gauchi, J. P.; Leveiller, F. J. Pharm. Sci., 2005, 94, 524.

[14] Di Martino, P.; Conflant, P.; Drache, M.; Huvenne, J. P.; GuyotHermann, A. M. J. Therm. Anal., 1997, 48, 447.

[15] Johari, G. P.; Kim, S.; Shanker, R. M. J. Pharm. Sci., 2005, 94, 2207.

[16] Nichols, G.; Frampton, C. S. J. Pharm. Sci., 1998, 87, 684.

[17] Burger, A.; Ramberger, R. Mikrochem. Acta, 1979, II, 273.

[18] Boldyreva, E.V.; Drebushchak, V.A.; Paukov, I.E.; Kovalevskaya, Y.A.; Drebushchak, T.N. J. Therm. Anal. Cal., 2004, 77, 607.

[19] Szelagiewicz, M.; Marcolli, C.; Cianferani, S.; Hard, A. P.; Vit, A.; Burkhard, A.; von Raumer, M.; Hofmeier, U. Ch.; Zilian, A.; Francotte, E.; Schenker, R. J. Therm. Anal. Calorim., 1999, 57, 23.

[20] Peterson, M. L.; Morissette, S. L.; McNulty, C.; Goldsweig, A.; Shaw, P.; LeQuesne, M.; Monagle, J.; Encina, N.; Marchionna, J.; Johnson, A.; Gonzalez-Zugasti, J.; Lemmo, A. V.; Ellis, S. J.; Cima, M. J.; Almarsson, O. J. Am. Chem. Soc., 2002, 124, 10958.

[21] Enke, D.; Janowski, F.; Schwieger, W. Microporous Mesoporous Mater., 2003, 60, 19.

[22] Janowski, F.; Enke, E. in: Handbook of Porous Solids (Eds: Schüth, F.; Sing, K.S.W.; Weitkamp, J.) Wiley-VCH: Weinheim, 2002, pp. 1432.

[23] Gopalakrishnan, T. R.; Beiner, M. Lett. Drug Des. Discov., 2006, 3, 723.

[24] Giordano, F.; Rossi, A.; Bettini, R.; Savioli, A.; Gazzaniga, A.; Novak, C. J. Therm. Anal. Cal., 2002, 68, 575 .

[25] Schönhals, A.; Göring, H.; Schick, C.; Zorn, R.; Frick, B. Coll. Polym. Sci., 2004, 282, 882 .

[26] Arndt, M.; Stannarius, R.; Groothues, H.; Hempel, E.; Kremer, F. Phys. Rev. Lett., 1997, 79, 2077.

[27] Alba-Simionesco, C.; Dosseh, G.; Dumont, E.; Frick, B.; Geil, B.; Morineau, D.; Teboul, V.; Xia, Y. Eur. Phys. J. E, 2003, 12, 19.

[28] Beiner, M.; Gopalakrishnan, T.R.; Pankaj, S.; Enke, D.; Steinhart, M. Nano Lett., 2007, 7, 1381.

[29] Avrami, M. J. Chem. Phys., 1939, 7, 1103; J. Chem. Phys., 1940, 8, 212.

[30] Turnbull, D.; Cormia, R.L. J. Chem. Phys., 1961, 34, 820.

[31] Reiter, G.; Castelein, G.; Sommer, J.-U.; Röttle, A.; Thurn-Albrecht, T. Phys. Rev. Lett., 2001, 87, 226101.

[32] Rengarajan, G.T. et al., to be published.

[33] Prasad, R.; Lele, S. Phil. Mag. Lett., 1994, 70, 357.

[34] Jackson, C.L.; McKenna, G.B. Chem. Mat., 1996, 8, 2128.

[35] Johari, G.P. Phil. Mag., 1998, 78, 375.

[36] Desgranges, C.; Delhommelle, J. J. Chem. Phys., 2007, 126, 054501.

[37] Ostwald, W. Z. Phys. Chem., 1897, 22, 289.

[38] Steinhart, M.; Goring, P.; Dernaika, H.; Prabhukaran, M.; Gösele, U.; Hempel, E.; Thurn-Albrecht, T. Phys. Rev. Lett., 2006, 97, 027801.

[39] Burley, J.C.; Duer, M.J.; Stein, R.S.; Vrcelj, R.M. Eur. J. Pharm. Sci., 2007, 31, 271. 